\title{
Relation of Neutrophil to Lymphocyte Ratio with Myocardial Damage in Patients Undergoing Elective Percutaneous Coronary Intervention
}

\author{
Farzad Emami' ${ }^{1}$ Fatemeh Pakrad $^{2, *}$, Jalal Poorolajal $^{3}$, Behshad Naghshtabrizi $^{4}$, \\ Hamidreza Golalikhani ${ }^{5}$, Azam Alizamir ${ }^{6}$ \\ ${ }^{1}$ Assistant Professor, Department of Cardiology, School of Medicine, Hamadan University of Medical Sciences, Hamadan, Iran \\ ${ }^{2}$ MSc in Nursing, School of Nursing and Midwifery, Hamadan University of Medical Sciences, Hamadan, Iran \\ ${ }^{3}$ Professor, Department of Epidemiology, School of Public Health, Hamadan University of Medical Sciences, Hamadan, Iran \\ ${ }^{4}$ Associate Professor, Department of Cardiology, School of Medicine, Hamadan University of Medical Sciences, Hamadan, Iran \\ ${ }^{5}$ Cardiologist, School of Medicine, Hamadan University of Medical Sciences, Hamadan, Iran \\ ${ }^{6}$ Assistant Professor, Department of Pathology, School of Medicine, Hamadan University of Medical Sciences, Hamadan, Iran
}

* Corresponding Author: Fatemeh Pakrad, School of Nursing and Midwifery, Hamadan University of Medical Sciences, Hamadan, Iran.Email: f_pakrad@yahoo.com

Received: 21.09 .2018

Accepted: 17.01 .2019

How to Cite this Article: Emami F, Pakrad F, Poorolajal J, Naghshtabrizi B, Golalikhani H, Alizamir A. Relation of Neutrophil to Lymphocyte Ratio with Myocardial Damage in Patients Undergoing Elective Percutaneous Coronary Intervention. Avicenna J Clin Med. 2019; 25(4): 185-192. DOI: 10.21859/ajcm.25.4.185

\section{Abstract}

Background and Objective: The high ratio of neutrophils to lymphocytes is a predictor of the risk of myocardial infarction in people who have coronary artery angiography. The purpose of this study was to measure this proportion in the assessment of damage through interventions in patients with non-emergency angioplasty.

Materials and Methods: A prospective cohort study was performed on 122 patients referring to Hamadan Farshchian Heart Center, Hamadan, Iran, during 2017 for selected angioplasty. Patients were divided into two groups according to the ratio of neutrophil/ lymphocyte. Data were collected through a checklist and blood test results, including neutrophil/lymphocyte ratio, erythrocyte sedimentation rate (ESR), C-reactive Protein (CRP), Creatine kinase-MB (CKMB), and troponin before angioplasty, as well as 6 and $12 \mathrm{~h}$ after angioplasty. The Chi-square test and independent t-test were performed using Stata software (version 14).

Results: There was no significant difference between the ESR levels in the two groups before the angioplasty $(\mathrm{P}=0.431)$. However, there were significant differences between the two groups $6 \mathrm{~h}(\mathrm{P}=0.001)$ and $12 \mathrm{~h}$ after the angioplasty $(\mathrm{P}=0.011)$. There was no significant difference between the blood level of troponin in both groups before $(\mathrm{P}=0.310)$ and $6 \mathrm{~h}$ after $(\mathrm{P}=0.186)$. However, there was a significant difference between the two groups $12 \mathrm{~h}$ after the angioplasty ( $\mathrm{P}=0.042)$.

Conclusion: The results showed that an increase in the level of troponin elevated neutrophil/lymphocyte ratio and ESR. However, there was no significant difference in CRP and CKMB levels, although there were significant changes in clinical status.

Keywords: Angioplasty, Lymphocyte, Myocardial Infarction, Neutrophil 


\section{بررسى ارتباط نسبت نوتروفيل به لنفوسيت با آسيب ميوكارد در بيماران كانديد}

\section{آنزّيويلاستى انتخابى}

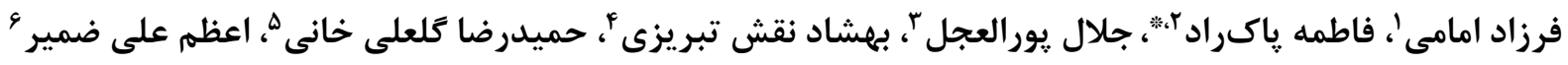

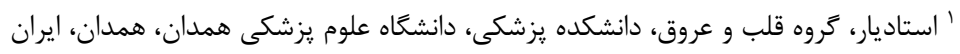

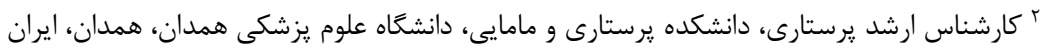

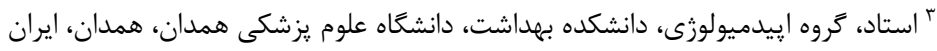

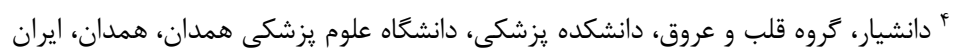

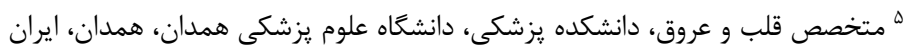

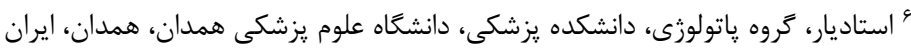
* نويسنده مسئول: فاطمه پاكراد، دانشكده يِرستارى و مامايى، دانشكاه علوم يزشكى همدان، همدان، ايران. ايميل: f_pakrad@yahoo.com

جكيده

سابقه و هدف: نسبت بالاى نوتروفيل به لنفوسيت (NLR: Neutrophil to Lymphocyte Ratio)

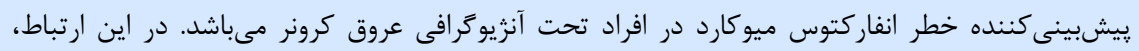

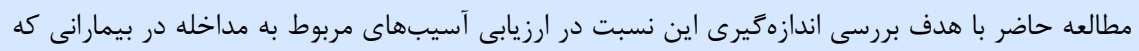

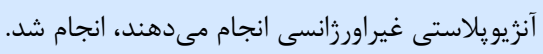

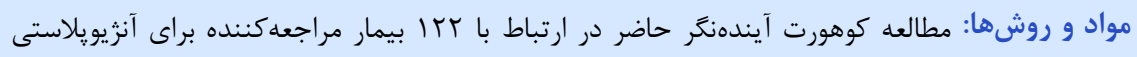

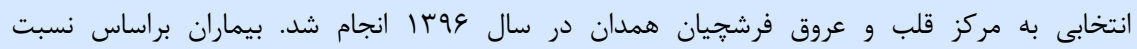

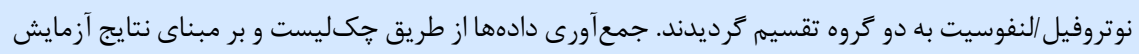

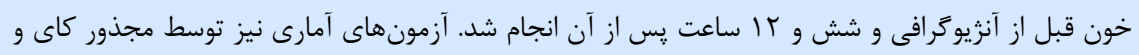

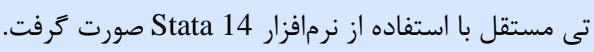

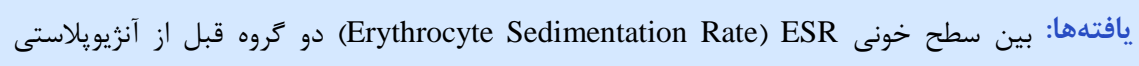

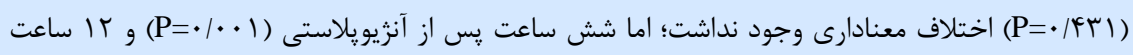

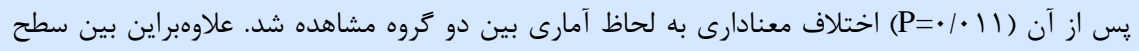

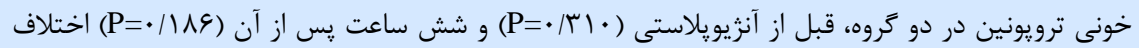

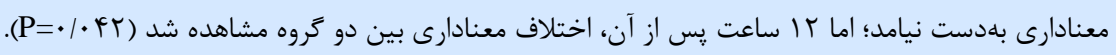

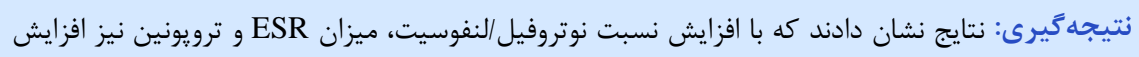

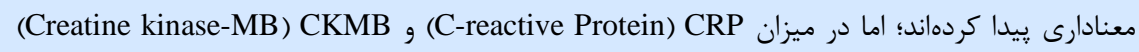

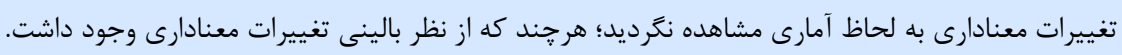

\section{وازًّان كليدى: آنزيويلاستى، انفاركتوس ميوكارد، لنفوسيت، نوتروفيل}

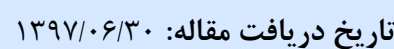

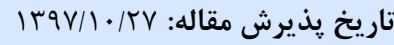
تمامى حقوق نشر براى دانشعاه علوم يزشكى همدان محفوظ است.
امكان يذير كرده است [1]]. يكى از شايعترين درمانهاى سكته

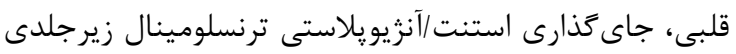

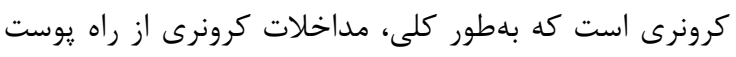
نامخذارى (PCI: Percutaneous Coronary Intervention) مىشود. استفاده از روش PCI موجب بهبود يِيش آكهى، تسكين

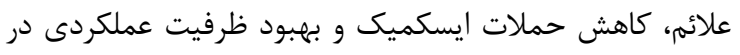

مقلدمه WHO: World Health ) سازمان جهانى بهداشت

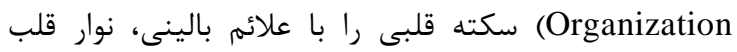

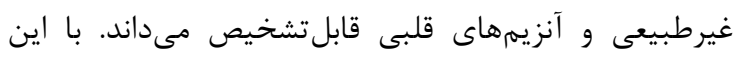

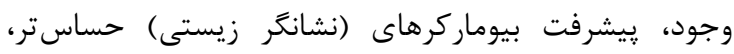
اختصاصى بافت قلب و ت تكنيكهاى تصويربردارى دقئ دقيق، تشخيص ميزان بسيار كم صدمه قلب و نكروز را در حال حاضر تصني 
يلاكتها علت اين حوادث باشند. نسبت نوتروفيل به لنفوسيت به

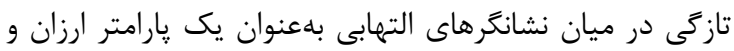

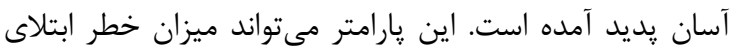

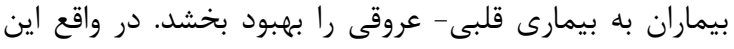

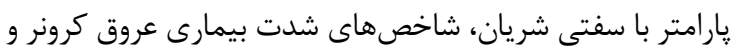

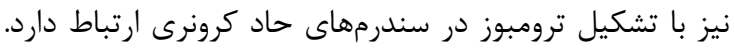

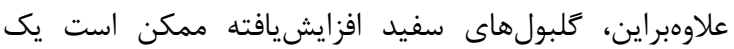

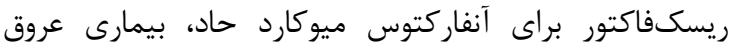

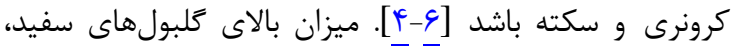

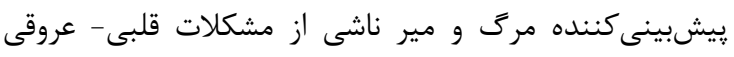

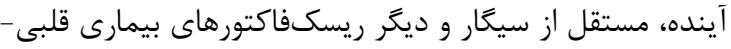

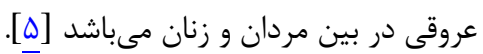

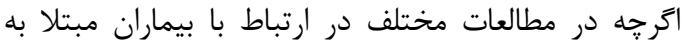

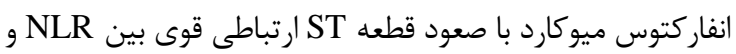

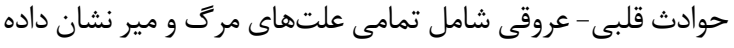

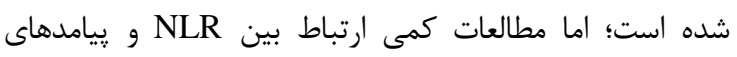
نامطلوب بالينى در بيماران تحت بازكردن عروق كرونرى المان

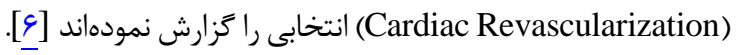
با توجه به موارد بيانشده، مطالعه حاضر به بررسى نسبت

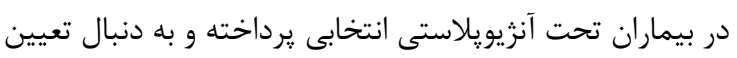
ميزان شدت سطح نوتروفيل به لنفوسيت با آسيب ميوكارد در دراني حين مداخله در اين بيماران مىباش نوفيل بهد.

\section{مواد و روشها}

مطالعه كوهورت آيندهنكر حاضر در مركز فوق تخصصى قلب

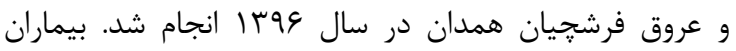

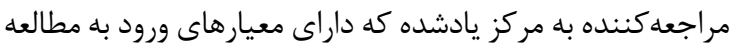

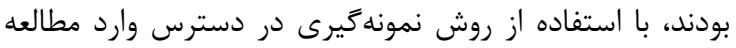

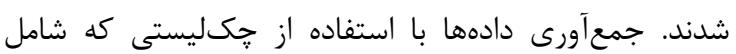

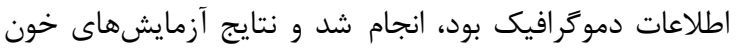

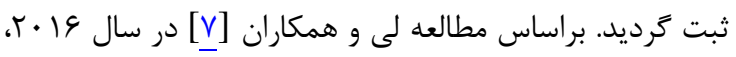

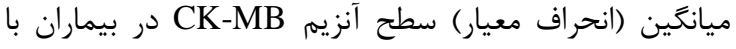

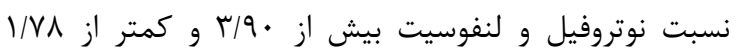

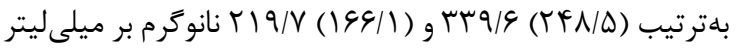
برآورد شده است. بر اين اساس، حجم نمونه در سطح اطمينان

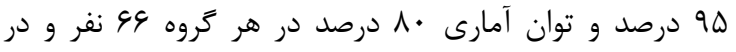

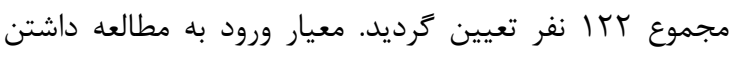

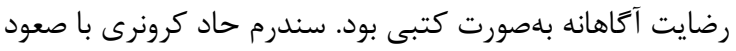

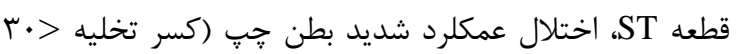

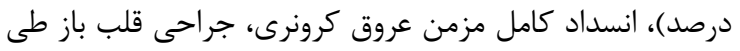

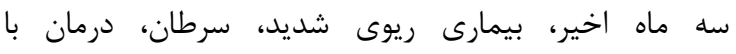

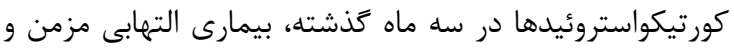

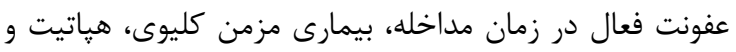
سكته مغزى نيز بهعنوان معيارهاى خروج در نظر كرفته شدند.
يروسيجرهاى نسبتاً كمخطر با روند بهبود سريع ميىشود.

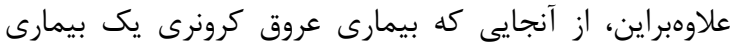

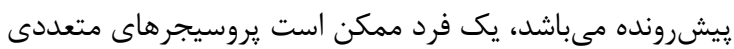

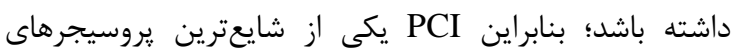

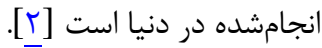
سندرمهاى حاد كرونرى و حوادث قلبى - مغزى اغلب اولين

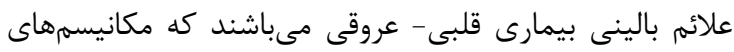

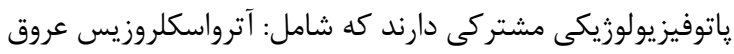

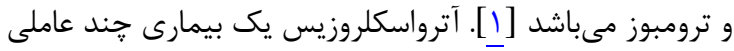

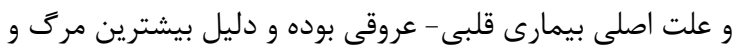

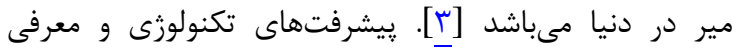

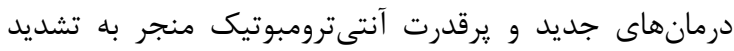

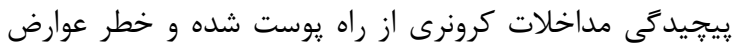

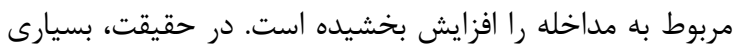

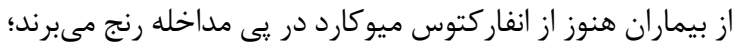

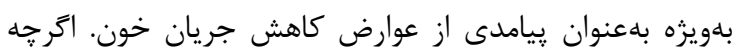

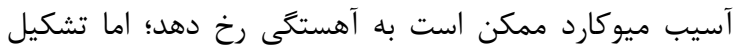

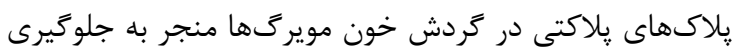

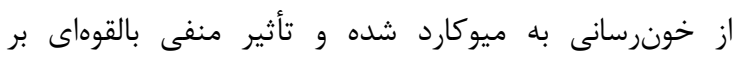

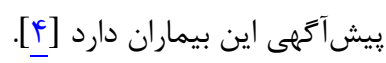
در مطالعات مختلف نقش التهاب در توسعه و وِيشرفت إندار

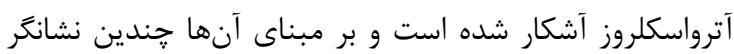

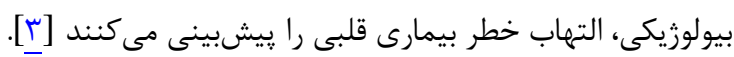

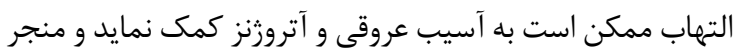

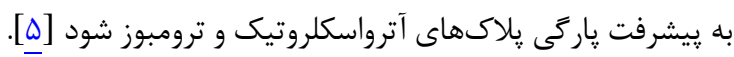

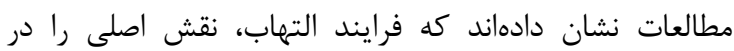

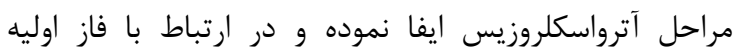

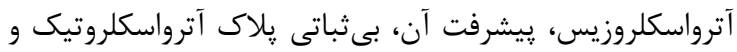

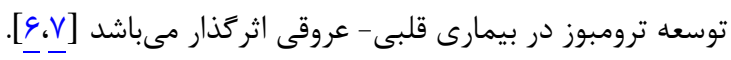

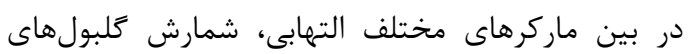

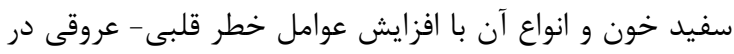

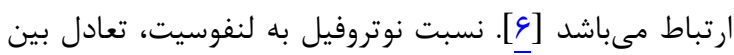

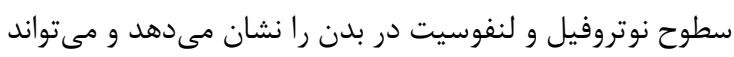

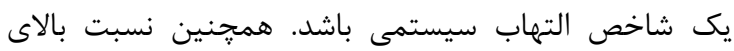

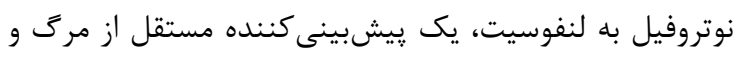

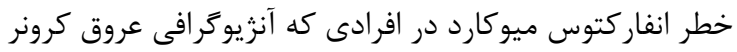

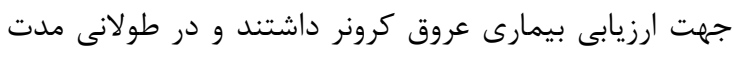

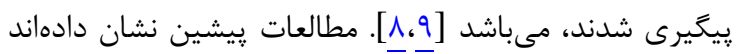

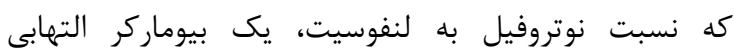

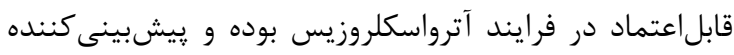

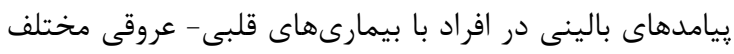

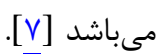

در حقيقت، كَلبولهاى سفيد خون و وضعيت التهابى

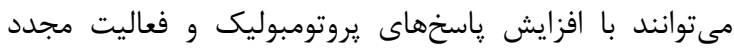


ماده ضد انعقاد موجود در لولههاى CBC، K3 EDTA بود. در اين مطالعه براى مقايسه متغيرهاى كيفى از آزمون مربع

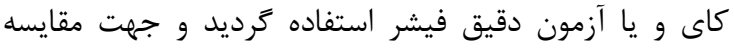

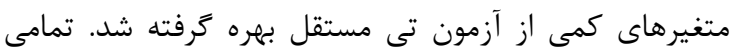

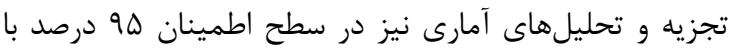

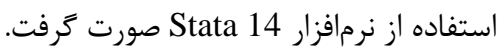

يافته ها در مطالعه حاضر بيشتر افراد مورد بررسى مردانى بودند كه

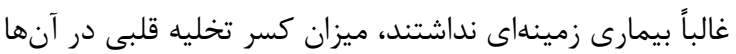

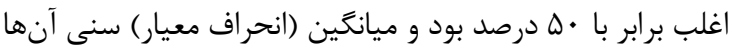

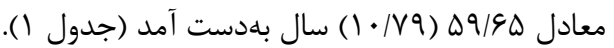

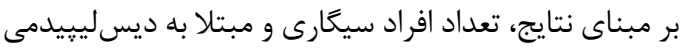

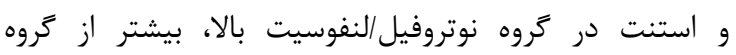

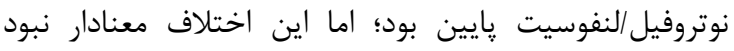

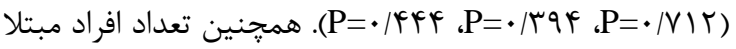

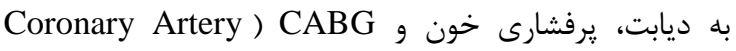
(Bypass Grafting

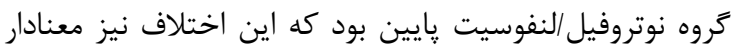

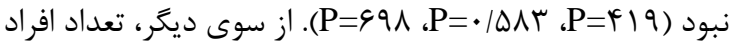
مبتلا به NSTEMI Elevation Myocardial ) NSTEMI Unstable Angina و در ₹روه با نسبت (Infarction نوتروفيل/لنفوسيت بالا، كمتر از گروه نوتروفيل/لنفوسيت پايين

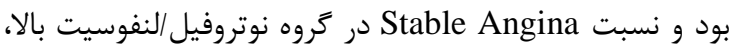

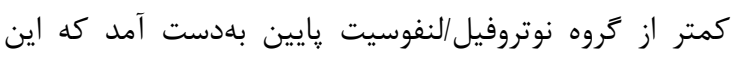

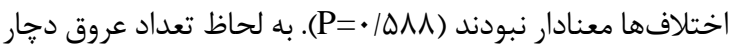

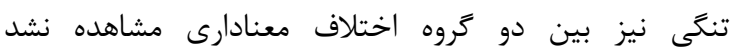

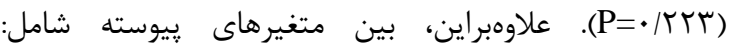
كلسترول، ترى گليسيريد، (Pow-density Lipoprotein) LDL) و و (High-density Lipoprotein) HDL اختلاف معنادارى بين

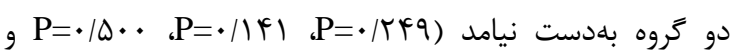

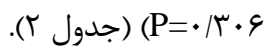

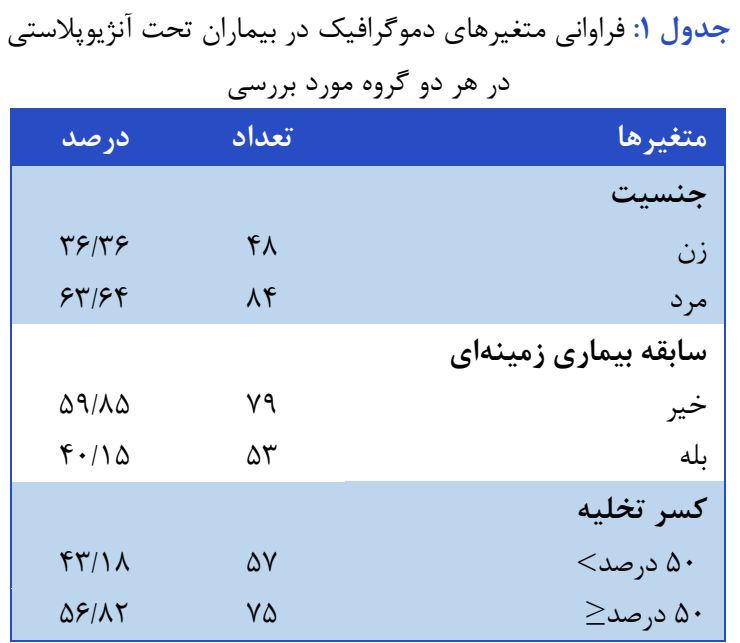

شايان ذكر است كه مواجهه مورد مطالعه، آسيب حين يروسيجر

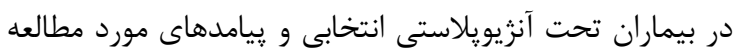

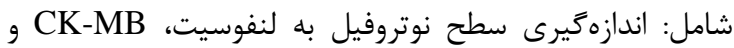

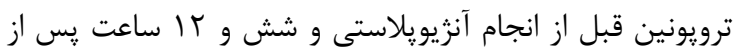

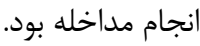
بايد خاطرنشان ساخت كه اين مطالعه به تأييد كميته اخلاق

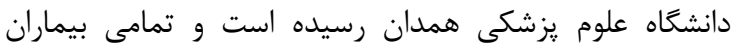

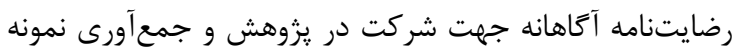

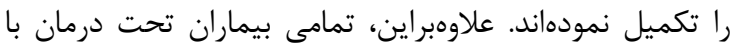

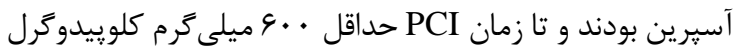

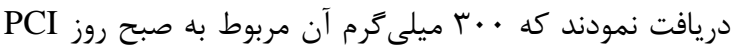

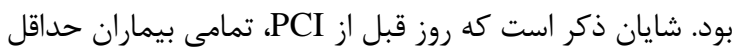

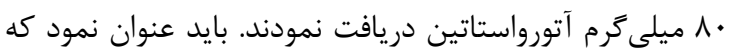

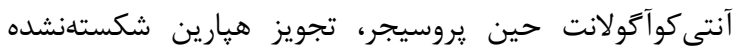

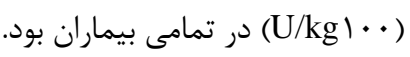

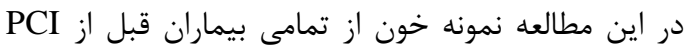

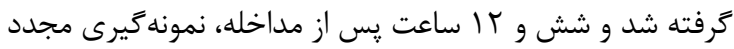

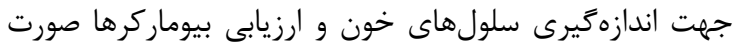

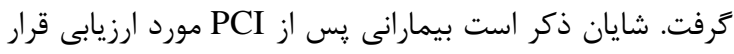

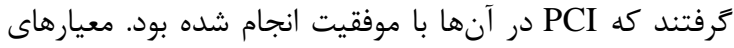

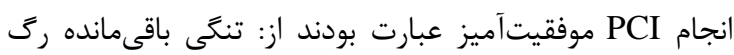
كرونر كمتر از • ب درصد و Thrombolysis in ) TIMI Myocardial Infarction

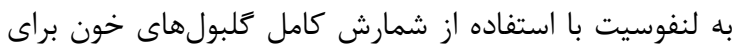

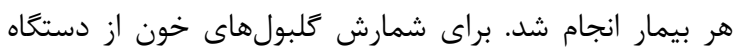
Sysmex kx-21 N كرديد. بررسى آسيب ميوكارد حول مداخله نيز با اندازهخيرى

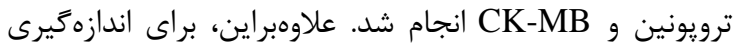

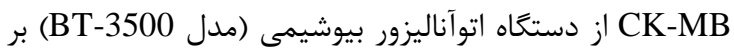

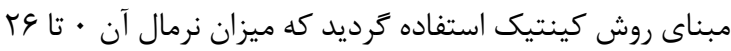
ميلى

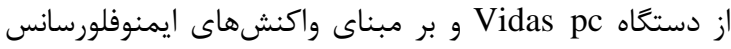
صورت كرفت. ترويونين بلهصورت كمى اندازمكيرى شد و ميزان

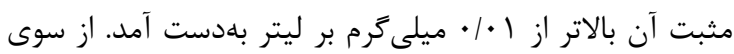

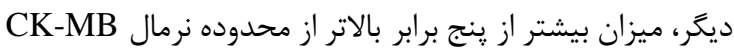

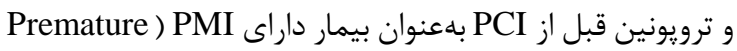
(Myocardial Infarction بيوماركرهاى قلبى به بيشتر از · r درصد از سطح پايه در

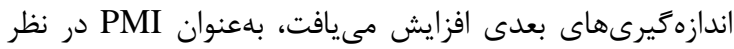

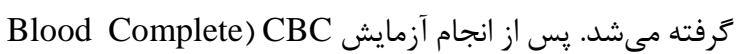

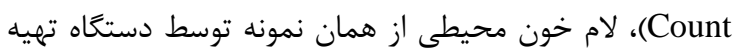

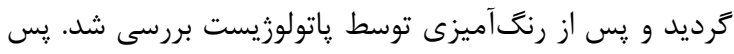

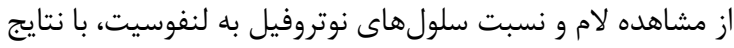

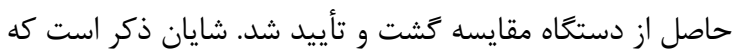




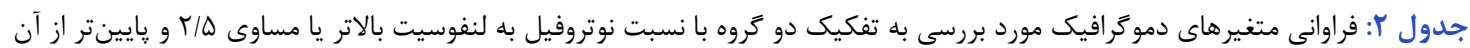

\begin{tabular}{|c|c|c|c|c|c|}
\hline \multirow{2}{*}{ سطح معنادارى } & \multicolumn{2}{|c|}{ كمتر از ه/ه } & \multicolumn{2}{|c|}{ بيشتر مساوى ب/ه ب } & نسبت نوتروفيل/لنفوسيت \\
\hline & درصد & تعداد & درصد & ت تعداد & متغير هاى كسسته \\
\hline \multirow{3}{*}{$\cdot / R I T$} & & & & & سيغار \\
\hline & $\varepsilon \Delta / 1 \Delta$ & r & $\varepsilon \wedge / 1 \wedge$ & Fa & خير \\
\hline & Tr/AD & זr & TI/AT & rI & بله \\
\hline \multirow{3}{*}{$\cdot / r q p$} & & & & & ديسليبيدمى \\
\hline & $V \Delta / V \varphi$ & $\Delta \cdot$ & AI/Ar & $\Delta F$ & خير \\
\hline & TF/TF & 19 & $1 \wedge / 11$ & ir & بله \\
\hline \multirow{3}{*}{.$/ 419$} & & & & & ديابت \\
\hline & AI/Ar & $\Delta F$ & $V V / T V$ & هI & خير \\
\hline & $\mid \wedge / 11$ & it & rt/R & 10 & بله \\
\hline \multirow{4}{*}{$\cdot / 0 \Lambda \Lambda$} & & & & & بيمارى قلبى \\
\hline & $\mid N / 1 \Lambda$ & it & TF/TF & 19 & سكته بدون بالارفتن قطعه ST \\
\hline & rV/A & ra & $r q / r q$ & rq & آنزين نايايدار \\
\hline & $\mathrm{Fr} / \mathrm{qF}$ & rq & re/rq & TY & آنزين پايدار \\
\hline \multirow{3}{*}{$\cdot / \Delta V Y$} & & & & & يرفشارى خون \\
\hline & VT/VT & FA & $99 \mid 9 \mathrm{~V}$ & FF & خير \\
\hline & TV/TV & 11 & 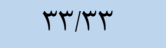 & trt & بله \\
\hline \multirow{3}{*}{.1991} & & & & & سابقه جراحى يِيوند عروق كرونرى \\
\hline & $94 / 9 \vee$ & st & $94 / 94$ & st & خير \\
\hline & $r / \cdot r$ & r & $91 \cdot 9$ & f & بله \\
\hline \multirow{5}{*}{ (trte } & & & & & تعداد عروق دجار تنكَى \\
\hline & $\cdot 1 \cdot$ & • & F/gr & r & s, \\
\hline & $\wedge \vee / \wedge \wedge$ & $\Delta \Lambda$ & $\Lambda \cdot / \cdot \cdot$ & $\Delta r$ & $\xi 1$ \\
\hline & $|r /| T$ & $\wedge$ & $\mid r / \Lambda \Delta$ & 9 & $\xi, r$ \\
\hline & $\cdot 1 \cdot \cdot$ & • & $1 / \Delta F$ & 1 & r \\
\hline \multirow{3}{*}{$\cdot$. FAF } & & & & & تعداد استنت \\
\hline & $\vee \wedge / \vee q$ & $\Delta T$ & 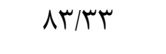 & $\Delta \Delta$ & 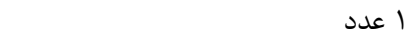 \\
\hline & $4 I / T)$ & 14 & $19 / 9 V$ & 11 & 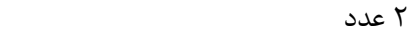 \\
\hline سطح معنادارى & انحراف معيار & ميانگين & انحراف معيار & ميانگين & متغير هاى بيوسته \\
\hline$\cdot / r \& q$ & rq/ar & $\mid \Delta T / 1 K$ & rN/Tr & $\mid F \Delta / \mu$ & كلسترول \\
\hline$\cdot|| F \mid$ & $94 / \pi \wedge$ & $19 Y / \Delta 9$ & $\Delta \Delta / \cdot r$ & $\mid F T / G D$ & ترى گليسيريد \\
\hline$\cdot 10 \cdots$ & 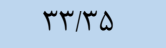 & 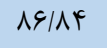 & TF/VT & 1r/rq & LDL \\
\hline$\cdot \pi \cdot \varphi$ & $r \Delta / \cdot r$ & $F r / \cdot r$ & $N / T^{K}$ & एN/दq & HDL \\
\hline
\end{tabular}

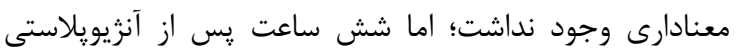

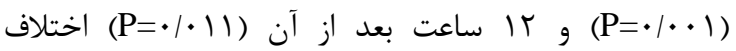

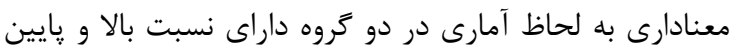

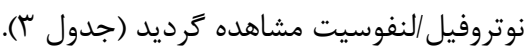
جدول \& أشان مى دهد كه بين سطح خونى ترويونين و نسبت

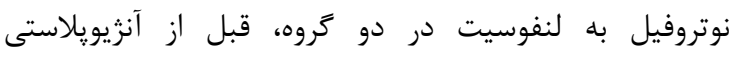

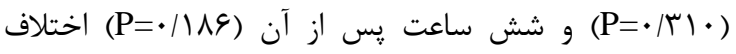

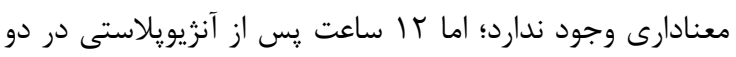

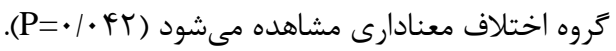

از سوى ديخر، بين سطح خونى CKMB با نسبت نوتروفيل

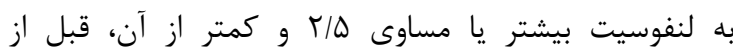

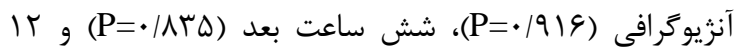

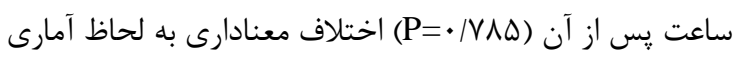

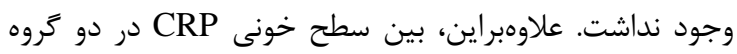

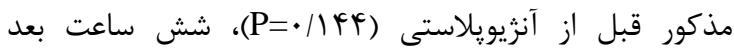
(P=•//\&9)

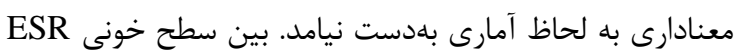

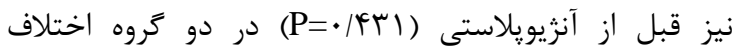




\begin{tabular}{|c|c|c|c|c|c|}
\hline \multirow[b]{2}{*}{ سطح معنادارى } & \multicolumn{2}{|c|}{ كمتر از ك/ه T } & \multicolumn{2}{|c|}{ بيشتر مساوى ه/ه } & \multirow{2}{*}{ نسبت نوتروفيل النفوسيت } \\
\hline & انحراف استاندارد & ميانكين & انحر اف استاندارد & ميانكين & \\
\hline & & & & & CKMP \\
\hline .1919 & 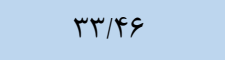 & rq/ब९ & rF/VT & $r \cdot / \Lambda$ & قبل از آنثيويلاستى \\
\hline - /ArL & $r \cdot / 4 \Delta$ & $r q / r$. & $r V / F q$ & TN/FT & شش ساعت پِ از آنزيويلاستى \\
\hline$\cdot / V \wedge \Delta$ & $r M / T$ & rN/GT & $|r / V|$ & $r V / V \cdot$ & זا ساعت پِ از آنزيويلاستى \\
\hline & & & & & ESR \\
\hline . & $I T / V$ & $\mid F / F \Delta$ & I./Ar & $|9| \cdot V$ & قبل از آنزيويلاستى \\
\hline$\cdot 1 \cdot \cdot 1$ & $N / \Delta K$ & $1 T / 94$ & $\mid T / \cdot F$ & $1 / 1 / 19$ & شش ساعت پِ از آنزيوِلاستى \\
\hline $.1 \cdot 11$ & $q / F V$ & $\mid r / \uparrow \wedge$ & IT/OT & NN/DT & ז' ساعت پِ از آنزيويلاستى \\
\hline & & & & & CRP \\
\hline.$/ 1 \mathrm{FF}$ & r/A & T/FT & (r/KG & $p / q$. & قبل از آنزيويلاستى \\
\hline .1199 & $\Gamma / 9 \Lambda$ & $r / T)$ & IG/AT & $\Delta / \wedge \vee$ & شش ساعت يس از آنزيوِلاستى \\
\hline - (1/r & $\Lambda / r \Lambda$ & $F / \cdot \varphi$ & $|V / \pi|$ & V/QT & זا ساعت پس از آنزيويلاستى \\
\hline
\end{tabular}

جدول F: ارتباط بين ترويونين در زمانهاى قبل، شش و rا ساعت پٍ از آنثيو يلاستى در دو گروه داراى نسبت نوتروفيل به لنفوسيت بيشتر يا مساوى

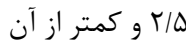

\begin{tabular}{|c|c|c|c|c|c|}
\hline \multirow{2}{*}{ سطح معنادارى } & \multicolumn{2}{|c|}{ كمتر از ه/ه } & \multicolumn{2}{|c|}{ بيشتر مساوى ه/ T } & نسبت نوتروفيل /لنفوسيت \\
\hline & درصد & تعداد & درصد & تعداد & متغير ها \\
\hline \multirow{3}{*}{$\cdot|r| \cdot$} & & & & & ترويونين قبل از آنزيويلاستى \\
\hline & 19/\%q & $\Delta 9$ & אז/או & $\Delta \Delta$ & خير \\
\hline & $1 \cdot|9|$ & v & $1919 \mathrm{~V}$ & 11 & بله \\
\hline \multirow{3}{*}{$\cdot / 1 \wedge 9$} & & & & & ترويونين شش ساعت يس از آنثيويلاستى \\
\hline & $\Lambda \cdot 19 \Lambda$ & VI & $V \cdot /$ is & r & خير \\
\hline & $19 / r 4$ & IV & $r q / \Delta \Delta$ & ir & بله \\
\hline \multirow{3}{*}{.$/ \cdot F t$} & & & & & ترويونين rا ساعت پِ از آنزيويلاستى \\
\hline & $\Lambda \cdot / r F$ & v. & $99 / \% 9$ & rF & 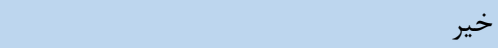 \\
\hline & $\mid \Delta / 9 \varphi$ & $1 \pi$ & $r \cdot|9|$ & 10 & بله \\
\hline
\end{tabular}

نوتروفيل/لنفوسيت در اين بيماران با بقاى ضعيف يس از جراحى

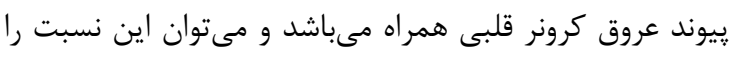

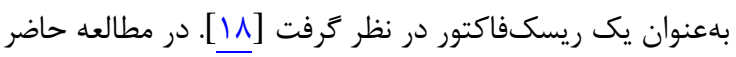

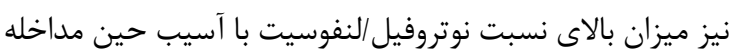
در ارتباط بود كه اين امر بيانكر بقاى ضعيفتر اين بيماران نسان نسبت

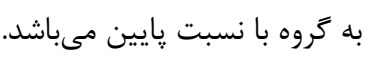

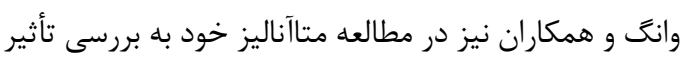

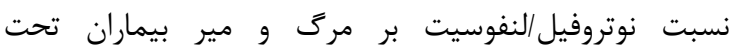

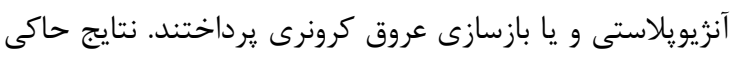

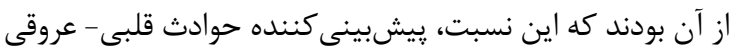

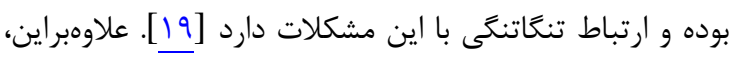

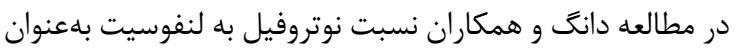

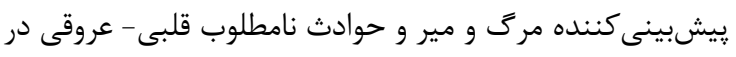
سندرم حاد كرونرى شناخته شد (·r). اين مسأله در مطالعات

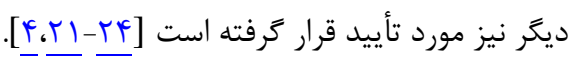

نتايج نشان دادند كه با افزايش نسبت نوتروفيل/لنفوسيت،

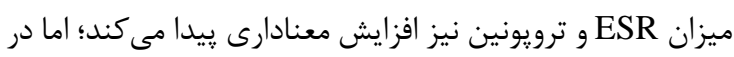

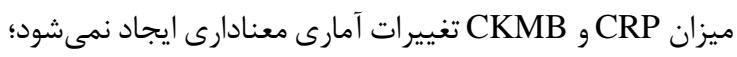
هرجند كه از نظر بالينى تغييرات معنادارى مشاهده ميى گردد.

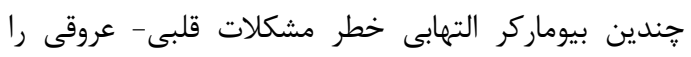

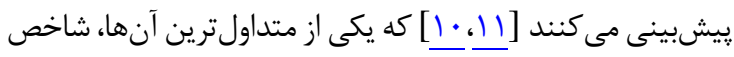

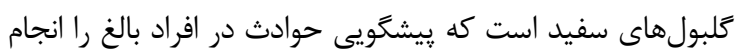

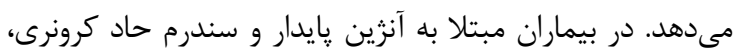

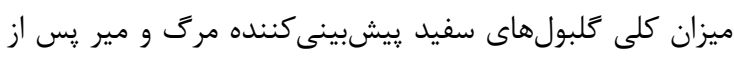

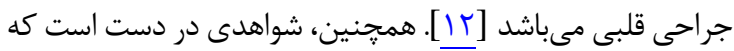

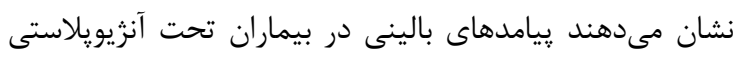

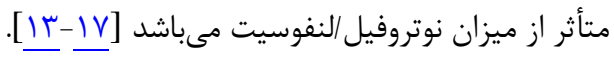

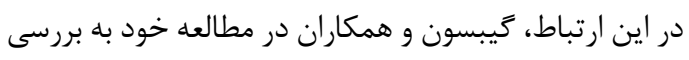

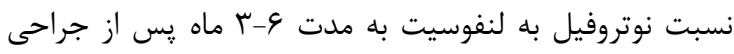

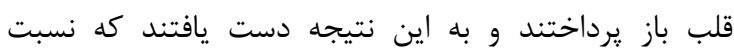


كرونرى يرداختند و گَزارش نمودند كه CRP توانايى يیشبينى

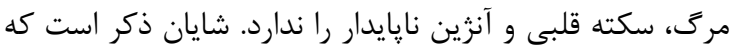

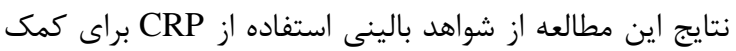

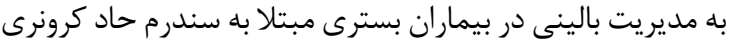

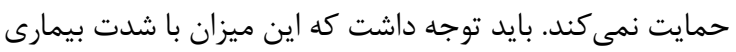

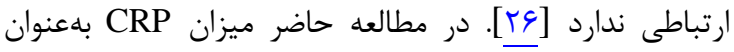

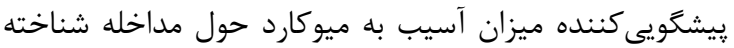

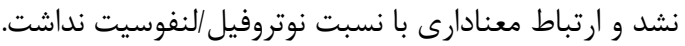

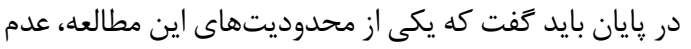

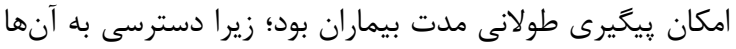

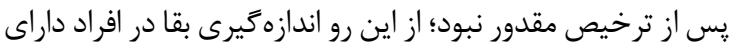
نسبت بالاى نوتروفيل/لنفوسيت انجام نشد. در اين راستا، يِيشنهاد مىشود پيگيرى اين بيماران در ساير محيطهاى درمانى فاقد

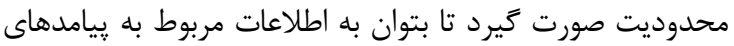
بالينى افزايش سطح اين آنزيمها در بيماران دست يافت بـت بـان.

نتيجه كيرى

بلهور كلى، نتايج نشان دادند كه بين سطح خونى بيماران

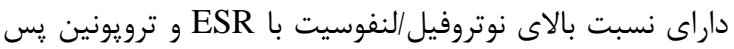

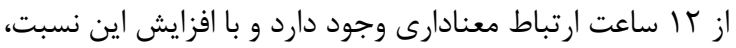
ميزان ESR و ترويونين نيز بيشتر مىشود. اين فاكتورها

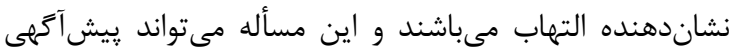

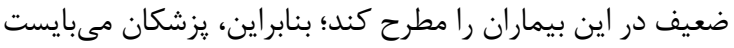

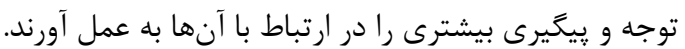

\section{تشكر و قلروراذى}

مقاله حاضر بركرفته از طرح تحقيقاتى مصوب دانشعاه علوم

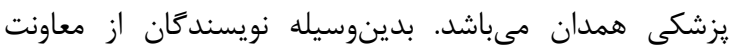
تحقيقات و فناورى و واحد توسعه تحقيقات بالينى بيمارستان

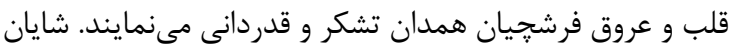

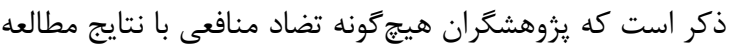
نداشتند.

\section{REFERENCES}

1. Mangiacapra F, Barbato E. Clinical implications of plateletvessel interaction. J Cardiovasc Transl Res. 2013;6(3):3105. PMID: 23271646 DOI: $10.1007 / s 12265-012-9441-0$

2. Trotter R, Gallagher R, Donoghue J. Anxiety in patients undergoing percutaneous coronary interventions. Heart Lung. 2011;40(3):185-92. PMID: 20723986 DOI: 10.1016/ j.hrtlng.2010.05.054

3. Karakas MS, Korucuk N, Tosun V, Altekin RE, Koç F, Ozbek SC, et al. Red cell distribution width and neutrophilto-lymphocyte ratio predict left ventricular dysfunction in acute anterior ST-segment elevation myocardial infarction. $J$ Saudi Heart Assoc. 2016;28(3):152-8. PMID: 27358532 DOI: 10.1016/j.jsha.2015.07.001

4. Verdoia M, Schaffer A, Barbieri L, Di Giovine G, Bellomo G, Marino P, et al. Impact of neutrophil-to-lymphocyte ratio on periprocedural myocardial infarction in patients undergoing non-urgent percutaneous coronary revascularisation.
در اين زمينه، ورديا و همكاران در مطالعه خود به بررسى اثر

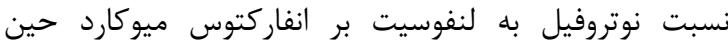

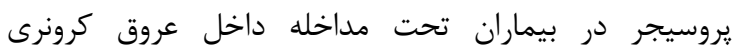

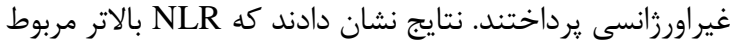

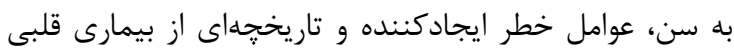
مىباشد. لازم به ذكر است كه در اين مطالعه نسبت نوتروفيل به ايها

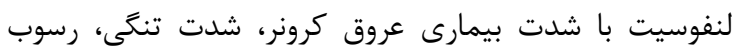

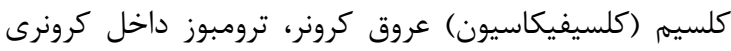

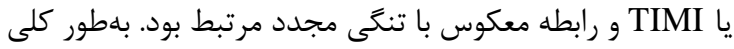

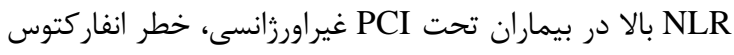

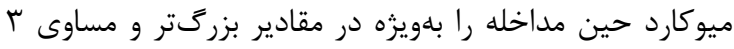

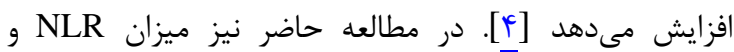

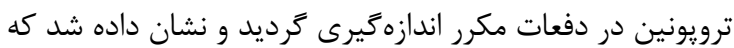

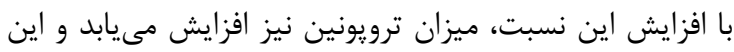
امر بيانكر آسيب حول مداخله مىبن باشد.

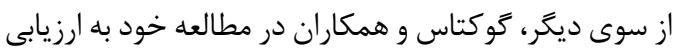

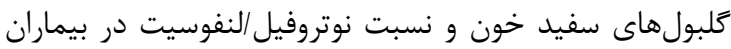

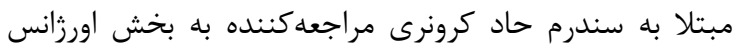

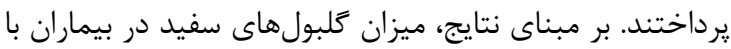

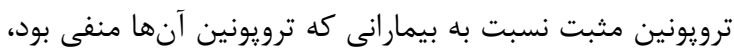

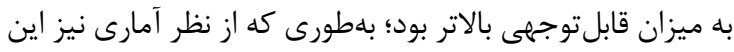

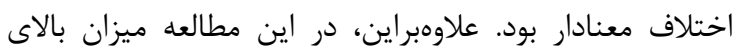

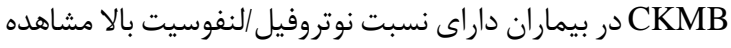
كرديد؛ به همين دليل، بيان شد كه اين نسبت در بدو بدون

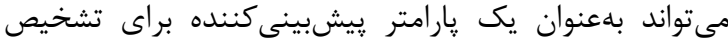

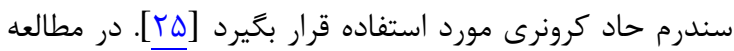

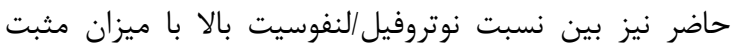

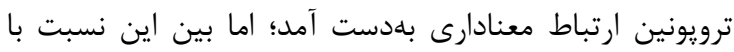

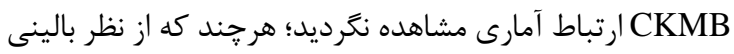
ارتباط وجود داشت.

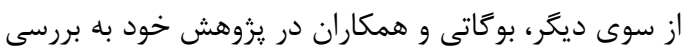

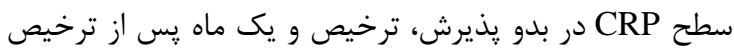

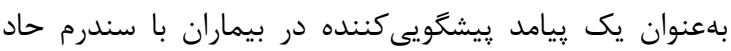

Neth Heart J. 2016;24(7):462-74. PMID: 27277659 DOI: 10.1007/s12471-016-0850-6

5. Brown DW, Giles WH, Croft JB. White blood cell count: an independent predictor of coronary heart disease mortality among a national cohort. J Clin Epidemiol. 2001;54(3):31622. PMID: 11223329

6. Cho KI, Ann SH, Singh GB, Her AY, Shin ES. Combined usefulness of the platelet-to-lymphocyte ratio and the neutrophil-to-lymphocyte ratio in predicting the long-term adverse events in patients who have undergone percutaneous coronary intervention with a drug-eluting stent. PLoS One. 2015;10(7):e0133934. PMID: 26207383 DOI: 10.1371/journal.pone.0133934

7. Lee MJ, Park SD, Kwon SW, Woo SI, Lee MD, Shin SH, et al. Relation between neutrophil-to-lymphocyte ratio and index of microcirculatory resistance in patients with stsegment elevation myocardial infarction undergoing 
primary percutaneous coronary intervention. Am J Cardiol. 2016;118(9):1323-8. PMID: 27600462 DOI: 10.1016/j. amjcard.2016.07.072

8. Ergelen M, Uyarel H, Altay S, Kul S, Ayhan E, Isik T, et al. Predictive value of elevated neutrophil to lymphocyte ratio in patients undergoing primary angioplasty for ST-segment elevation myocardial infarction. Clin Appl Thromb Hemost. 2014;20(4):427-32. PMID: 23314674 DOI: 10.1177/ 1076029612473516

9. Akpek M, Kaya MG, Lam YY, Sahin O, Elcik D, Celik T, et al. Relation of neutrophil/lymphocyte ratio to coronary flow to in-hospital major adverse cardiac events in patients with ST-elevated myocardial infarction undergoing primary coronary intervention. Am J Cardiol. 2012;110(5):621-7. PMID: 22608360 DOI: 10.1016/j.amjcard.2012.04.041

10. Danesh J, Collins R, Appleby P, Peto R. Association of fibrinogen, C-reactive protein, albumin, or leukocyte count with coronary heart disease: meta-analyses of prospective studies. JAMA. 1998;279(18):1477-82. PMID: 9600484

11. Pearson TA, Mensah GA, Alexander RW, Anderson JL, Cannon RO 3rd, Criqui M, et al. Markers of inflammation and cardiovascular disease: application to clinical and public health practice: a statement for healthcare professionals from the centers for disease control and prevention and the American Heart Association. Circulation. 2003;107(3):499511. PMID: 12551878

12. Hajj-Ali R, Zareba W, Ezzeddine R, Moss AJ. Relation of the leukocyte count to recurrent cardiac events in stable patients after acute myocardial infarction. Am J Cardiol. 2001; 88(11):1221-4. PMID: 11728346

13. Poludasu S, Cavusoglu E, Khan W, Marmur JD. Neutrophil to lymphocyte ratio as a predictor of long-term mortality in African Americans undergoing percutaneous coronary intervention. Clin Cardiol. 2009;32(12):E6-10. PMID: 20014207 DOI: 10.1002/clc.20503

14. Shen XH, Chen Q, Shi Y, Li HW. Association of neutrophil/lymphocyte ratio with long-term mortality after ST elevation myocardial infarction treated with primary percutaneous coronary intervention. Chin Med J (Engl). 2010;123(23):3438-43. PMID: 22166528

15. Park JJ, Jang HJ, Oh IY, Yoon CH, Suh JW, Cho YS, et al. Prognostic value of neutrophil to lymphocyte ratio in patients presenting with ST-elevation myocardial infarction undergoing primary percutaneous coronary intervention. Am J Cardiol. 2013;111(5):636-42. PMID: 23273716 DOI: 10.1016/j.amjcard.2012.11.012

16. Soylu K, Yuksel S, Gulel O, Erbay AR, Meric M, Zengin $\mathrm{H}$, et al. The relationship of coronary flow to neutrophil/lymphocyte ratio in patients undergoing primary percutaneous coronary intervention. $J$ Thorac Dis. 2013;5(3):258-64. PMID: 23825756 DOI: 10.3978/j.issn. 2072-1439.2013.05.13

17. Sen N, Afsar B, Ozcan F, Buyukkaya E, Isleyen A, Akcay
$\mathrm{AB}$, et al. The neutrophil to lymphocyte ratio was associated with impaired myocardial perfusion and long term adverse outcome in patients with ST-elevated myocardial infarction undergoing primary coronary intervention. Atherosclerosis. 2013;228(1):203-10. PMID: 23489347 DOI: 10.1016/j.atherosclerosis.2013.02.017

18. Gibson PH, Croal BL, Cuthbertson BH, Small GR, Ifezulike AI, Gibson G, et al. Preoperative neutrophil-lymphocyte ratio and outcome from coronary artery bypass grafting. Am Heart J. 2007;154(5):995-1002. PMID: 17967611 DOI: 10.1016/j.ahj.2007.06.043

19. Wang X, Zhang G, Jiang X, Zhu H, Lu Z, Xu L. Neutrophil to lymphocyte ratio in relation to risk of all-cause mortality and cardiovascular events among patients undergoing angiography or cardiac revascularization: a meta-analysis of observational studies. Atherosclerosis. 2014;234(1):206-13. PMID: 24681815 DOI: 10.1016/j.atherosclerosis.2014.03.003

20. Dong CH, Wang ZM, Chen SY. Neutrophil to lymphocyte ratio predict mortality and major adverse cardiac events in acute coronary syndrome: a systematic review and metaanalysis. Clin Biochem. 2018;52:131-6. PMID: 29132766 DOI: 10.1016/j.clinbiochem.2017.11.008

21. Fowler AJ, Agha RA. Neutrophil/lymphocyte ratio is related to the severity of coronary artery disease and clinical outcome in patients undergoing angiography--the growing versatility of NLR. Atherosclerosis. 2013;228(1):44-5. PMID: 23474126 DOI: 10.1016/j.atherosclerosis.2013.02.008

22. Nakazawa KR, Wengerter SP, Power JR, Lookstein RA, Tadros RO, Ting W, et al. Preoperative inflammatory status as a predictor of primary patency after femoropopliteal stent implantation. J Vasc Surg. 2017;66(1):151-9. PMID: 28259571 DOI: 10.1016/j.jvs.2017.01.031

23. Soylu K, Gedikli O, Dagasan G, Aydin E, Aksan G, Nar G, et al. Neutrophil-to-lymphocyte ratio predicts coronary artery lesion complexity and mortality after non-ST-segment elevation acute coronary syndrome. Rev Port Cardiol. 2015;34(7-8):465-71. PMID: 26164277 DOI: 10.1016/j. repc. 2015.01 .013

24. Montecucco F, Liberale L, Bonaventura A, Vecchiè A, Dallegri F, Carbone F. The role of inflammation in cardiovascular outcome. Curr Atheroscler Rep. 2017; 19(3):11. PMID: 28194569 DOI: 10.1007/s11883-017-0646-1

25. Göktaş MU, Akyol PY, Karakaya Z, Payza U, Topal FE. Evaluation of white blood cell and neutrophil/lymphocyte ratio in acute coronary syndrome patients admitted to emergency department. Biomed Res. 2018;29(10):2009-14.

26. Bogaty P, Boyer L, Simard S, Dauwe F, Dupuis R, Verret B, et al. Clinical utility of c-reactive protein measured at admission, hospital discharge, and 1 month later to predict outcome in patients with acute coronary disease: the risca (recurrence and inflammation in the acute coronary syndromes) study. J Am Coll Cardiol. 2008;51(24):2339-46. PMID: 18549920 DOI: 10.1016/j.jacc.2008.03.019 